\title{
Measurement of the Promoter Activity in Escherichia coli by Using a Luciferase Reporter
}

\author{
Yuki Yamanaka ${ }^{1,2, \$}$, Hiroki Watanabe ${ }^{1}$, Erika Yamauchi ${ }^{1}$, \\ Yukari Miyake ${ }^{1}$ and Kaneyoshi Yamamoto, 2, *
}

\begin{abstract}
${ }^{1}$ Department of Frontier Bioscience, Hosei University, Tokyo, Japan; ${ }^{2}$ Micro-Nano Technology Research Center, Hosei University, Tokyo, Japan; ${ }^{\$}$ Current/Present address: Mechanobiology Institute, National University of Singapore, Singapore, Singapore

*For correspondence: kanyamam@hosei.ac.jp
\end{abstract}

\begin{abstract}
[Abstract] The reporter system is widely used technique for measuring promoter activity in bacterial cells. Until now, a number of reporter system have been developed, but the bioluminescent reporter constructed from the bacterial luciferase genes is one of the useful systems for measuring in vivo dynamics of gene expression. The introduced bioluciferase lux reporter enables easy, fast, and sensitive measurement of the promoter activity without cell lysis because the substrates of bioluminescent reaction are synthesized inside the bacterial cell, thereby allowing low-cost experiments. This protocol describes a high throughput technique to measure the promoter activity in Escherichia coli K-12 using the lux reporter system.
\end{abstract}

Keywords: Iux operon, Luciferase, Escherichia coli, Promoter activity, High-throughput assay, H-NS silencer

[Background] The promoter activity in vivo was measured using a reporter system such as the lac $Z$ (encoding $\beta$-galactosidase), gus (encoding $\beta$-gulucuronidase), and cat (encoding chloramphenicol acetyltransferase) genes. In the case of the lacZ reporter system, for instance, the test promoter sequence is fused to a promoter-less lac $Z$ gene, creating a test promoter-lac $Z$ fusion gene, which is then transferred into a recipient cell. For the measurement of the activity of the test promoter, however, the whole cell lysate must be prepared to detect in vitro $\beta$-galactosidase activity by adding a substrate such as ONPG (O-Nitrophenyl- $\beta$-D-galactopyranoside). To avoid such biochemical procedures, the fluorescent gfp gene, coding green fluorescent protein (GFP), was employed as a reporter which can be detected without cell lysis. Thus, the fluorescent reporter system is more convenient than the systems which requires measurement of enzymatic activity. However, the fluorescent proteins have a technical limitation especially in genes that are expressed at low levels because of high background noise that arises from intrinsic autofluorescence of cells. To overcome this problem, the luminescent reporter has been developed, which catalyzes bioluminescence reactions using the substrate as luciferins (Meighen, 1991). The Photorhabdus luminescens bioluminescence luxCDABE genes, coding two luciferase subunits (LuXAB) and three proteins (LuXCDE), which are important for substrate biosynthesis (Bjarnason et al., 2003). Once the test promoter is fused to promoter-less luxCDABE, both luciferase and its substrate are expressed under the control of the test promoter, and the promoter activity can be easily determined by measuring luminescence without the cell lysis (Bjarnason et al., 2003). This 
bioluminescent reporter system is recognized as a powerful high-throughput assay for studying continuous kinetics of promoter activity (Yamanaka et al., 2018; Burton et al., 2010). In this protocol, we describe how to construct the bioluminescent reporter system and how to measure the promoter activity in E. coli.

\section{Materials and Reagents}

1. Pipette tips (Thermo Fisher Scientific, catalog number: QSP Liquid Handling Products $110-\mathrm{Q}$ and 111-Q) (Gilson, catalog number: DIAMOND Tips DL10 and D5000)

2. $1.5 \mathrm{ml}$ plastic tube (Rikaken, catalog number: STAR MicroTestTube $1.5 \mathrm{ml}$ RSV-MTT1.5)

3. $0.22 \mu \mathrm{m}$ filter (Advantec, catalog number: 13CP020AS)

4. Glass tubes (Iwaki, catalog number: TEST18NP)

5. Sterile $50 \mathrm{ml}$ plastic tube (Iwaki, catalog number: 2345-050)

6. BD Falcon 96-well plates, Black/Clear BD Optilux (Becton Dickinson, catalog number: 353948)

7. 96-well white plate (Becton Dickinson, catalog number: 353377)

8. Petri dishes (Rikaken, catalog number: STAR SDish9015 ver.2 RSU-SD9015-2)

9. pLUX vector (Burton et al., 2010)

10. Specific primers (Thermo Fisher Scientific, Custom DNA Oligos) (Table 1)

11. TaKaRa Ex Taq (Takara Bio, catalog number: RR001A)

12. NucleoSpin Gel and PCR Clean-up (Macherey-Nagel, catalog number: 740609.10)

13. Restriction enzyme Xhol (Takara Bio, catalog number: 1094)

14. Restriction enzyme Bam HI (Takara Bio, catalog number: 1010)

15. In-Fusion HD Cloning Plus (Takara Bio, catalog number: 638920)

16. Kanamycin Monosulfate (Nacalai Tesque, catalog number: 19839-44)

17. Plasmid DNA Extraction Mini Kit (Favorgen, catalog number: FAPDE 001)

18. BigDye ${ }^{\circledR}$ Terminator v3.1 Ready Reaction Mix (Applied Biosystems, catalog number:4337455)

19. IPTG (Nacalai Tesque, catalog number: 06289-67)

20. $3 \mathrm{M}$ sodium acetate (Nacalai Tesque, catalog number: 06893-24)

21. Ethanol (Nacalai Tesque, catalog number: 14710-25)

22. $\mathrm{Hi}-\mathrm{Di}^{\mathrm{TM}}$ Formamide (Applied Biosystems, catalog number: 4311320)

23. Bacto ${ }^{\mathrm{TM}}$ tryptone (BD Biosciences, catalog number: 211705 )

24. Bacto ${ }^{\mathrm{TM}}$ yeast extract (BD Biosciences, catalog number: 212750),

25. $\mathrm{NaCl}$ (Nacalai Tesque, catalog number: 31320-05)

26. $\mathrm{NaOH}$ (Nacalai Tesque, catalog number: 31511-05)

27. $\mathrm{Na}_{2} \mathrm{HPO}_{4} \cdot 12 \mathrm{H}_{2} \mathrm{O}$ (Nacalai Tesque, catalog number: 31722-45)

28. $\mathrm{KH}_{2} \mathrm{PO}_{4}$ (Wako, catalog number: 498748161612)

29. $\mathrm{MgCl}_{2} \cdot 6 \mathrm{H}_{2} \mathrm{O}$ (Nacalai Tesque, catalog number: 20908-65)

30. $\mathrm{K}_{2} \mathrm{SO}_{4} \cdot 12 \mathrm{H}_{2} \mathrm{O}$ (Nacalai Tesque, catalog number: 01727-25)

31. $\mathrm{NH}_{4} \mathrm{Cl}_{2}$ (Nacalai Tesque, catalog number: 02424-55) 
32. $\mathrm{CaCl}_{2}$ (Nacalai Tesque, catalog number: 08894-25)

33. D-(+)-Glucose (Nacalai Tesque, catalog number: 16805-35)

34. 0.5 M EDTA (Nacalai Tesque, catalog number: 06894-14)

35. Competent E. coli DH5a, provided from National Institute of Genetics in Japan (preparation at time of use) (see Recipes)

36. $50 \mathrm{mg} / \mathrm{ml}$ kanamycin (see Recipes)

37. LB broth (see Recipes)

38. LB agar with $50 \mu \mathrm{g} / \mathrm{ml}$ kanamycin (see Recipes)

39. $125 \mathrm{mM}$ EDTA (see Recipes)

40. $70 \%$ ethanol (see Recipes)

41. M9-Glucose medium (see Recipes)

\section{Equipment}

1. Pipettes (Gilson, models: PIPETMAN P2, P10, P20, P100, P200, P1000, P5000)

2. Centrifuge (Tomy, model: MX-301)

3. Thermal Cycler (Applied Biosystems, model: 2720Thermal Cycler)

4. Temperature chamber (Taitec, model: Thermo minder SM-10R)

5. Water bath shaker (Taitec, model: Personal-11)

6. DNA sequencer (Applied Biosystems, model: 3500Genetyc Analyzer)

7. Plate reader (Corona, model: MTP-880Lab)

8. Autoclave (Tomy Seiko, model: LSX-500)

\section{Software}

1. SF6 for Windows (Corona, in only Japanese)

2. Microsoft Excel (Microsoft)

\section{Procedure}

A. Construction of pLUX reporter plasmids

1. Determine promoter region around a target gene. Typically, we take a region of DNA from 500 base-pairs (bp) upstream to $150 \mathrm{bp}$ downstream of distal transcription start site (see for instance, the determined promoters of 18 target genes Figure 1). 


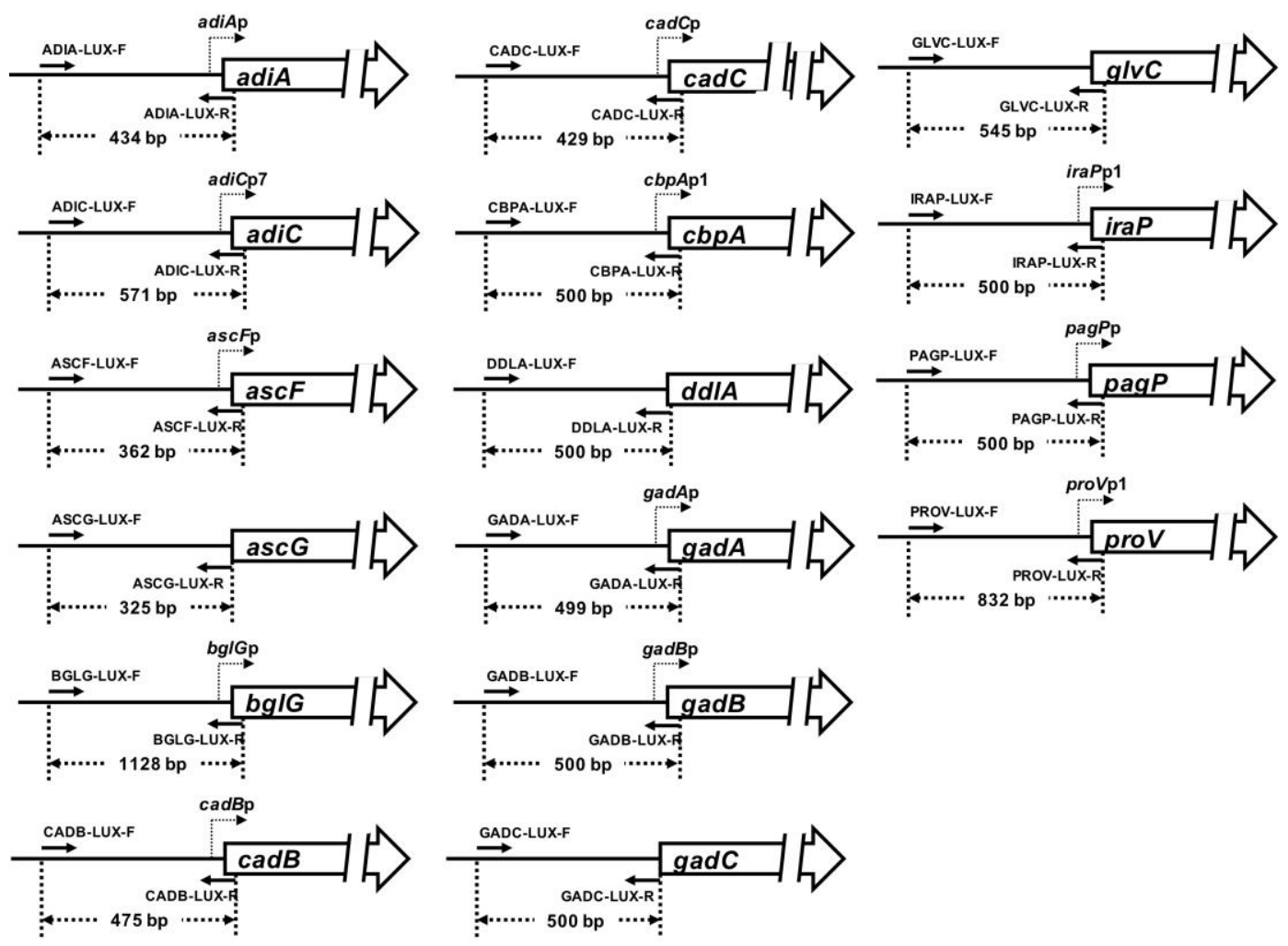

Figure 1. Eighteen E. coli promoter regions into pLux

2. Design primers which including 15 overlapping nucleotides (nt) with the pLUX vector for amplification of the determined promoter region (see Figure 2 and Table 1).

3. Amplify target promoter regions by PCR using Ex Taq polymerase (Takara Bio). Standard reaction condition is used as described in the manual for Ex Taq polymerase (Takara Bio). In most of the cases, thermal cycle conditions are as follows: after heating at $98{ }^{\circ} \mathrm{C}$ for $5 \mathrm{~min}$, process 30 cycles of $98^{\circ} \mathrm{C}$ for $10 \mathrm{sec}$ (denature), $50^{\circ} \mathrm{C}$ for $30 \mathrm{sec}$ (annealing), and $72{ }^{\circ} \mathrm{C}$ for 1 min (elongation) (Figure 2).

4. Purify the PCR product by NucleoSpin Gel and PCR Clean-up (Macherey-Nagel).

Note: When PCR product includes non-specific bands, the target product is cut from agarose gel after electrophoresis with total volume of PCR product and then purified by NucleoSpin Gel and PCR Clean-up (Macherey-Nagel).

5. Digest pLUX vector using restriction enzymes $X$ ho I and Bam $\mathrm{HI}$, and then purify the liner pLUX by NucleoSpin Gel and PCR Clean-up (Macherey-Nagel).

Note: For cloning into $p L U X$, we recommend the double-digestion of $p L U X$ by both Xho I and Bam HI (Figure 2).

6. Clone a target promoter as the purified PCR product into the liner pLUX using In-Fusion HD Cloning Plus (Takara Bio).

Note: We recommend in vivo E. coli cloning (iVEC) as an alternative DNA cloning method (Nozaki et al., 2019), which is provided from National BioResource Project (NBRP) of Japan.

7. Add a part $(\sim 10 \mu \mathrm{l})$ of the In-Fusion reaction mixture into $0.1 \mathrm{ml}$ of the suspension of competent 
E. coli DH5a (see Recipes), and then incubate the mixture on ice for $30 \mathrm{~min}$.

8. Heat the mixture at $42^{\circ} \mathrm{C}$ for $45 \mathrm{sec}$, and immediately add $0.9 \mathrm{ml}$ of $\mathrm{LB}$ broth to the mixture.

9. Incubate $1 \mathrm{ml}$ of the suspension of $E$. coli DH5a transformants at $37^{\circ} \mathrm{C}$ for $60 \mathrm{~min}$.

10. Spread $0.1-0.2 \mathrm{ml}$ of the suspension of $E$. coli DH5a transformants onto the LB agar with 50 $\mu \mathrm{g} / \mathrm{ml}$ kanamycin, and then incubate the agar plate at $37^{\circ} \mathrm{C}$ overnight.

11. Isolate a transformant of $E$. coli $\mathrm{DH} 5 \alpha$ harboring the cloned candidates as a single colony on the LB agar with $50 \mu \mathrm{g} / \mathrm{ml}$ kanamycin.

12. Inoculate a single colony into $5 \mathrm{ml}$ of LB broth containing $0.05 \mathrm{ml}$ of $50 \mathrm{mg} / \mathrm{ml}$ kanamycin in a glass tube (Iwaki), and incubate the culture in water bath (Taitec) at $37^{\circ} \mathrm{C}$ with shaking (120 rpm) overnight.

13. Isolate the plasmid from the transformant cells of $5 \mathrm{ml}$ overnight culture by Plasmid DNA Extraction Mini Kit (Favorgen).

14. Sanger reaction is performed with the isolated plasmid as a template, LUX-R primer (Table 1), and BigDye ${ }^{\circledR}$ Terminator v3.1 Ready Reaction Mix (Applied Biosystems) according to the recommended procedure by Supplier.

15. Transfer $20 \mu \mathrm{l}$ of sanger product into a sterile $1.5 \mathrm{ml}$ plastic tube and then add $2 \mu \mathrm{l}$ of $125 \mathrm{mM}$ EDTA, $2 \mu \mathrm{l}$ of $3 \mathrm{M}$ sodium acetate, and $50 \mu \mathrm{l}$ of ethanol (Nacalai Tesque).

16. Mix the solution by vortex mixer for $15 \mathrm{sec}$.

17. Collect DNA pellet by centrifugation ( $\left.17,800 \times \mathrm{g}, 4^{\circ} \mathrm{C}, 15 \mathrm{~min}\right)$.

18. Add $70 \mu \mathrm{l}$ of $70 \%$ ethanol and collect DNA pellet by centrifugation $\left(17,800 \times \mathrm{g}, 4^{\circ} \mathrm{C}, 15 \mathrm{~min}\right)$.

19. Dissolve DNA pellet by $15 \mu \mathrm{l}$ of $\mathrm{Hi}-\mathrm{Di}^{\mathrm{TM}}$ Formamide (Applied Biosystems).

20. Determine DNA sequence of the promoter region cloned into the isolated plasmid with DNA sequencer (Applied Biosystems). 
1. Amplification of the test promoter by PCR

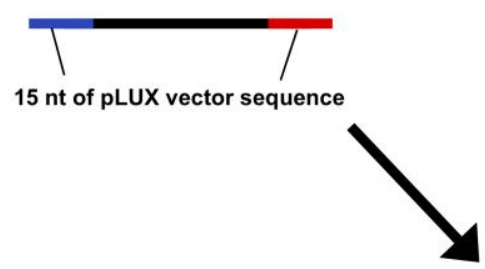

2. Digestion of pLUX vector by Xho I and Bam HI

3. Insertion of PCR product into pLUX vector using In-Fusion enzyme

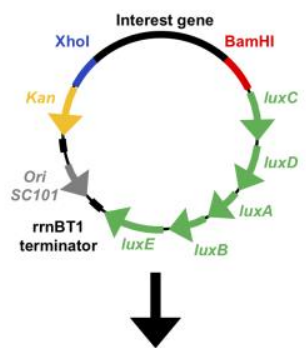

4. Transformation of DH5 $\alpha$

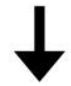

5. Isolation of the plasmid and confirmation of the inserted promoter sequence by DNA sequencer

Figure 2. Strategy for construction of pLUX reporter plasmid. The promoter region was amplified by PCR using the E. coli K-12 genome as the template and a pair of specific primers (Table 1). The amplified DNA fragment was inserted into a pLUX vector using the In-Fusion HD cloning kit (Takara Bio). After transformation of $\mathrm{DH} 5 \alpha$, the plasmid was purified from a culture of transformant cells. The DNA sequence of insertion on the resulting plasmids was confirmed by DNA sequencing. 
Table 1. The used primers and pLUX derivatives

\begin{tabular}{|c|c|c|}
\hline Name & Characterization & Reference \\
\hline \multicolumn{3}{|l|}{ Primers } \\
\hline ADIA-LUX-F & 5'- TCGTCTTCACCTCGAATGGGGATATTCCAGCGGGTCATGC -3' (for amplification of adiA promoter) & This study \\
\hline ADIA-LUX-R & 5'- ACTAACTAGAGGATCCATTGCTTACCCGGTTATGAAGGAA -3' (for amplification of adiA promoter) & This study \\
\hline ADIC-LUX-F & 5'- TCGTCTTCACCTCGAGAAACTGAGTCAGAAAAGGAACGAA -3' (for amplification of adiC promoter) & This study \\
\hline ADIC-LUX-R & 5'- ACTAACTAGAGGATCAATTAAACTCCTGCGAAGGCGAGCT -3' (for amplification of adiC promoter) & This study \\
\hline ASCF-LUX-F & 5'- TCGTCTTCACCTCGAGCACGCGGGAAACGGTCGCTTTTGA -3' (for amplification of ascF promoter) & This study \\
\hline ASCF-LUX-R & 5'- ACTAACTAGAGGATCCTATCACCGAGCGTGCCAGCGCCGC -3' (for amplification of ascF promoter) & This study \\
\hline ASCG-LUX-F & 5'- TCGTCTTCACCTCGAGATGTTATCAACGCCGCCCAGTGCC -3' (for amplification of ascG promoter) & This study \\
\hline ASCG-LUX-R & 5'- ACTAACTAGAGGATCCCCCCGGCGCGCTTCGCCACTTCCAG -3' (for amplification of ascG promoter) & This study \\
\hline BGLG-LUX-F & 5'- TCGTCTTCACCTCGAGACAAATAATTCACCAGACA -3' (for amplification of bg/G promoter) & This study \\
\hline BGLG-LUX-R & 5'- ACTAACTAGAGGATCGTGTTCTTTGCGCACGCGCT -3' (for amplification of $b g / G$ promoter) & This study \\
\hline CADB-LUX-F & 5'- TCGTCTTCACCTCGATTTAATTTACGCCCAGGGGCAAACA -3' (for amplification of cadB promoter) & This study \\
\hline CADB-LUX-R & 5'- ACTAACTAGAGGATCGCTCTTCTCCTAATTTCATTTTTGA -3' (for amplification of cadB promoter) & This study \\
\hline CADC-LUX-F & 5'- TCGTCTTCACCTCGACGTGCGGCCCCGTGATGCTGTTGAA -3' (for amplification of cadC promoter) & This study \\
\hline CADC-LUX-R & 5'- ACTAACTAGAGGATCAATAGAAACTCATTCGAAAAGGGAA -3' (for amplification of cadC promoter) & This study \\
\hline CBPA-LUX-F & 5'- TCGTCTTCACCTCGAATTATCATTCTGCATTTCCTCAAAT -3' (for amplification of cbpA promoter) & This study \\
\hline CBPA-LUX-R & 5'- ACTAACTAGAGGATCAGCGTTATCTCGCGTAAATCAACAC -3' (for amplification of cbpA promoter) & This study \\
\hline DDLA-LUX-F & 5'- TCGTCTTCACCTCGAGCAAGCTTAAATAACAACTCAGCAA -3' (for amplification of ddIA promoter) & This study \\
\hline DDLA-LUX-R & 5'- ACTAACTAGAGGATCCTTAAAAACCTATCCCGTCTAACAC -3' (for amplification of ddIA promoter) & This study \\
\hline GADA-LUX-F & 5'- TCGTCTTCACCTCGAGAAAAAAGACTTTAACTTTGGGGAA -3' (for amplification of gadA promoter) & This study \\
\hline GADA-LUX-R & 5'- ACTAACTAGAGGATCTTCGAACTCCTTAAATTTATTTGAA -3' (for amplification of gadA promoter) & This study \\
\hline GADB-LUX-F & 5'- TCGTCTTCACCTCGATCAATATGACGATCCTGCAGC -3' (for amplification of gadB promoter) & This study \\
\hline GADB-LUX-R & 5'- ACTAACTAGAGGATCTTTAAACTCCTTAAAATGAT -3' (for amplification of gadB promoter) & This study \\
\hline GADC-LUX-F & 5'- TCGTCTTCACCTCGACTGGCGGATGAAATCGCCAAACTGG -3' (for amplification of gadC promoter) & This study \\
\hline GADC-LUX-R & 5'- ACTAACTAGAGGATCATTATCCCCCTAAAACGGTATTCCT -3' (for amplification of gadC promoter) & This study \\
\hline GLVC-LUX-F & 5'- TCGTCTTCACCTCGAGCGCACCAGGTAAGTGCCACTACCG -3' (for amplification of $g / v C$ promoter) & This study \\
\hline GLVC-LUX-R & 5'- ACTAACTAGAGGATCGCACTGGCGTGAACATCGCGCCGCC -3' (for amplification of $g / v C$ promoter) & This study \\
\hline IRAP-LUX-F & 5'- TCGTCTTCACCTCGATTACCACCAAAAACGATTCCTACCC -3' (for amplification of iraP promoter) & This study \\
\hline IRAP-LUX-R & 5'- ACTAACTAGAGGATCGTCTGTATTTCCTTATCCAAAGTAT -3' (for amplification of iraP promoter) & This study \\
\hline PAGP-LUX-F & 5'- TCGTCTTCACCTCGAGCTGATTAAAATCAAGAAAAACTGC -3' (for amplification of pagP promoter) & This study \\
\hline PAGP-LUX-R & 5'- ACTAACTAGAGGATCTTGTGACCATAAAACATTTATCAAA -3' (for amplification of pagP promoter) & This study \\
\hline PROV-LUX-F & 5'- TCGTCTTCACCTCGAATCTCTGGGACAACGTGAAG -3' (for amplification of proV promoter) & This study \\
\hline PROV-LUX-R & 5'- ACTAACTAGAGGATCCCAGACTGGCGTCTTTTACG -3' (for amplification of proV promoter) & This study \\
\hline Lux-R & 5'-GGCAGGTAAACACTATTATCACC-3' & $\begin{array}{l}\text { Yamanaka } \\
\text { et al., } 2014\end{array}$ \\
\hline \multicolumn{3}{|l|}{ pLUX } \\
\hline \multicolumn{3}{|l|}{ derivatives } \\
\hline pLUX & promoter-less luxCDABE & $\begin{array}{l}\text { Burton et } \\
\text { al., } 2010\end{array}$ \\
\hline pLUXgadWp & pLUX, gadW'-lux & $\begin{array}{l}\text { Burton et } \\
\text { al., } 2010\end{array}$ \\
\hline
\end{tabular}




\begin{tabular}{|c|c|c|}
\hline pLUXslpp & pLUX, s/p'-Iux & $\begin{array}{l}\text { Burton et } \\
\text { al., } 2010\end{array}$ \\
\hline pLUXadiAp & pLUX, adiA-lux (the adiA promotert [ $434 \mathrm{bp}$ ] is -399 to +35 from transcription start site of adiAp) & This study \\
\hline pLUXadiCp & pLUX, adiC-Iux (the adiC promotert [ $571 \mathrm{bp}]$ is -400 to +171 from transcription start site of adiCp7) & This study \\
\hline pLUXascFp & pLUX, ascF-lux (the ascF promotert [ $362 \mathrm{bp}]$ is -228 to +134 from transcription start site of ascFp) & This study \\
\hline pLUXascGp & pLUX, ascG-lux (the ascG promotert [325 bp] is -325 to -1 from transcription start site of ascGp) & This study \\
\hline pLUXbglGp & pLUX, bg/G-Iux (the $b g / G$ promotert [1128 bp] is -164 to +964 from transcription start site of $b g / G p$ ) & This study \\
\hline pLUXcadBp & pLUX, cadB-lux (the $c a d B$ promotert [ $475 \mathrm{bp}$ ] is -400 to +75 from transcription start site of $c a d B p$ ) & This study \\
\hline pLUXcadCp & pLUX, cadC-lux (the cadC promotert [ $429 \mathrm{bp}$ ] is -400 to +29 from transcription start site of $c a d C p$ ) & This study \\
\hline pLUXcbpAp & pLUX, $c b p A-I u x$ (the $c b p A$ promotert [500 bp] is -442 to +58 from transcription start site of $c b p A p 1$ ) & This study \\
\hline pLUXddlAp & $\begin{array}{l}\text { pLUX, ddlA-lux (the ddIA promotert [500 bp] is }-500 \text { to }-1 \text { from the fist nucleotide of translation start codon of } \\
d d l A \text { ) }\end{array}$ & This study \\
\hline pLUXgadAp & pLUX, gadA-lux (the gadA promotert [ $499 \mathrm{bp}$ ] is -472 to +27 from transcription start site of gadAp) & This study \\
\hline pLUXgadBp & pLUX, gadB-lux (the gadB promotert [500 bp] is -473 to +27 from transcription start site of gadBp) & This study \\
\hline pLUXgadCp & $\begin{array}{l}\text { pLUX, gadC-lux (the gadC promotert [500 bp] is }-500 \text { to }-1 \text { from the fist nucleotide of translation start codon } \\
\text { of } g a d C \text { ) }\end{array}$ & This study \\
\hline pLUXglvCp & $\begin{array}{l}\text { pLUX, } g / v C \text {-lux (the } g / v C \text { promotert [ } 545 \mathrm{bp}] \text { is }-496 \text { to }+49 \text { from the fist nucleotide of translation start codon } \\
\text { of } g / v C \text { ) }\end{array}$ & This study \\
\hline pLUXiraPp & pLUX, iraP-lux (the iraP promotert [500 bp] is -433 to +67 from transcription start site of iraPp1) & This study \\
\hline pLUXpagPp & pLUX, pagP-lux (the pagP promotert [500 bp] is -469 to +31 from transcription start site of pagPp) & This study \\
\hline pLUXproVp & pLUX, proV-Iux (the proV promotert [832 bp] is -432 to +400 from transcription start site of proVp1) & This study \\
\hline
\end{tabular}


B. Measurement of luciferase activity in E. coli

1. Transform E. coli strains by the cloned luciferase reporter plasmids.

Note: The $\Delta h n s \Delta h h a \Delta y d g T$ strain, isolated from E. coli K-12 W3110 strain, deleted three genes, hns, hha, and ydgT, in its genome (Ueda et al., 2013). H-NS plays a role in transcriptional silencing of genes, which is modulated by Hha and YdgT proteins in E. coli. The $\Delta h n s \Delta h h a \Delta y d g T$ strain was transformed with pQE80L, pQE80Lhns which carries hns gene in pQE80L, pQE80Lhns-I70A which carries hns gene with substitutions of lle70Ala, or pQE80Lhns-L75A which carries hns gene with substitutions of Leu75Ala. These transformants were used as hosts for luciferase measurements.

2. Inoculate at least three single colonies of $E$. coli transformant in glass tubes separately under M9-glucose medium including $50 \mu \mathrm{g} / \mathrm{ml}$ kanamycin.

Note: In addition of kanamycin, ampicillin should be added in medium at the final concentration of $100 \mu \mathrm{g} / \mathrm{ml}$ for the $\triangle \mathrm{hns} \triangle \mathrm{hha} \triangle \mathrm{ydg} T$ strain harboring a $\mathrm{pQE} 80 \mathrm{~L}$ derivative and a pLux derivative.

3. Incubate the pre-cultures at $37^{\circ} \mathrm{C}$ with shaking for overnight.

4. Inoculate $100 \mu \mathrm{l}$ of overnight pre-culture in $10 \mathrm{ml}$ of fresh M9-glucose medium including $50 \mu \mathrm{g} /$ $\mathrm{ml}$ kanamycin.

5. Incubate the $10 \mathrm{ml}$ cultures at $37^{\circ} \mathrm{C}$ in water bath (Taitec) with shaking (120 rpm) until luciferase activity is measured.

6. Transfer $100 \mu \mathrm{l}$ of culture to a well of a Black/Clear BD Falcon 96-well plate (Becton Dickinson) in triplicate for each culture.

Note: We tested 96-well plates for measuring luminescence in E. coli with the plate reader (Corona), indicating that the 96-well white plate showed the leakage of $1.5 \%$ to an adjacent empty well whereas the 96 -well black plate reduced leakage by $0.95 \%$ (see the row data in both 96 plates in Figure 3). Although a white plate is usually used for luminescent measurements, a black plate was used to prevent leakage of high luminescence from one culture to other well in this study. Additionally, during measurement we arranged cultures with a single well gap between them to minimize leakage to adjacent wells. However, we recommend that a white plate be used first as standard procedure. 
Please cite this article as: Yamanaka et. al., (2020). Measurement of the Promoter Activity in Escherichia coli by Using a Luciferase Reporter,Bio-protocol

1. The measured intensity of luciferase activity in E. coli

in 96 well black plate (Becton Dickinson, catalog number: 353948)

\begin{tabular}{|l|r|r|r|r|r|r|r|r|r|r|r|r|r|r|}
\hline & 1 & 2 & 3 & 4 & 5 & 6 & 7 & 8 & 9 & 10 & & 11 & 12 \\
\hline A & 136,932 & 178,982 & 140,691 & 517 & & & & & & & & \\
\hline B & 924 & 959 & 792 & -239 & & & & & & & & \\
\hline C & & & & & & & & & & & & \\
\hline D & & & & & & & & & & & & \\
\hline E & & & & & & & & & & & & \\
\hline F & & & & & & & & & & & & \\
\hline G & & & & & & & & & & & & \\
\hline H & & & & & -375 & -268 & -220 & & -383 & -77 & -205 & \\
\hline
\end{tabular}

2. The measured intensity of luciferase activity in $E$. coli

in 96 well white plate (Becton Dickinson, catalog number: 353377)

\begin{tabular}{|l|r|r|r|r|r|r|r|r|r|r|r|r|r|}
\hline & 1 & 2 & 3 & 4 & 5 & 6 & 7 & 8 & 9 & 10 & & 11 & 12 \\
\hline A & 363,077 & 473,103 & 359,535 & 23,512 & & & & & & & & \\
\hline B & 19,944 & 20,826 & 19,223 & 14,633 & & & & & & & & \\
\hline C & & & & & & & & & & & & \\
\hline D & & & & & & & & & & & & \\
\hline E & & & & & & & & & & & & \\
\hline F & & & & & & & & & & & & \\
\hline G & & & & & 14,720 & 14,231 & 14,500 & & 13,816 & 13,617 & 13,094 & \\
\hline H & & & & & & & & & & & & & \\
\hline
\end{tabular}

Figure 3. The measured intensity of luciferase activity in E. coli in 96 well plates. E. coli K-12 W3110 (parent strain) harbouring pLUXappYp, containing appY-lux operon (data not shown) was grown in M9-glucose medium at $37^{\circ} \mathrm{C}$, and culture was applied into three wells (A1, A2, and A3) of both the 96-well black plate (Becton Dickinson, catalog number: 353948) (upper) and the 96-well white plate (Becton Dickinson, catalog number: 353377) (lower). M9glucose medium ( $\mathrm{H} 5, \mathrm{H} 6$, and $\mathrm{H} 7)$ and distilled water $(\mathrm{H} 9, \mathrm{H} 10$, and $\mathrm{H} 11)$ were also applied into three wells of both the 96 well plates. Each intensity is shown as a raw data measured with the plate reader (Corona). The fluorescent intensity was detected in the empty wells adjacent to wells filled by culture (A4 and B1 to B4).

7. Transfer $100 \mu \mathrm{l}$ of fresh M9-glucose medium to another well of the Black/Clear BD Falcon 96 well plate (Becton Dickinson) used in Step B6 in triplicate for background.

8. Set the Black/Clear BD Falcon 96 well plate (Becton Dickinson) containing the samples and the background with plate reader (Corona).

9. Measure $\mathrm{OD}_{600}$ according to the procedure for the plate reader (Corona).

10. Measure luminescence as a total intensity. Therefore, no filter is set according to the procedure for the plate reader (Corona).

\section{Data analysis}

1. Extract raw numeric data of both the values of $O D_{600}$ and the intensities of luminescence as a text file from SF6 for Windows (Corona).

2. Open a text file containing the raw numeric data in Microsoft Excel (Microsoft).

3. Normalize the net values of $\mathrm{OD}_{600}$ and the net intensities of luminescence with background.

4. Calculate the ratio of luminescence to $\mathrm{OD}_{600}$ as specific activity of the promoter of each culture by the following formula: the net intensity of luminescence/the net values of $\mathrm{OD}_{600}$. 
5. Average the ratios of triplicate with standard deviation.

Note: The activities of the 18 promoters used are represented in Figure 4, indicating that all 18 promoters silenced by H-NS were de-silenced by two H-NS mutants in agreement with our previous work (Yamanaka et al., 2018). To confirm a significant difference, calculated p-values by $t$-test of statistical analysis should be evaluated at less than 0.01 .

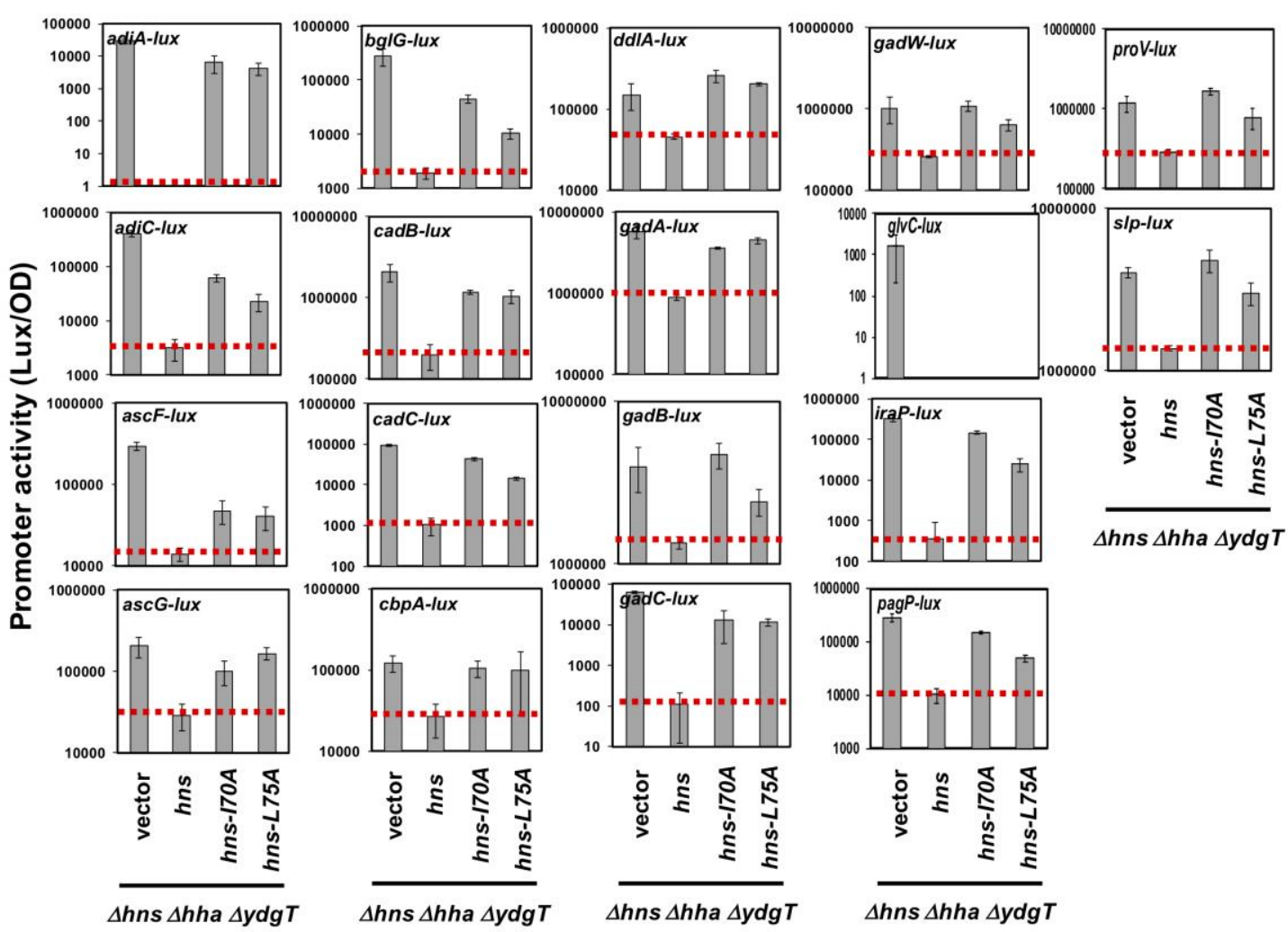

Figure 4. Luciferase reporter reveals silence of 18 promoters by H-NS in E. coli. The constructed 18 pLux reporter plasmids, pLUXadiAp, pLUXadiCp, pLUXascFp, pLUXascGp, pLUXbglGp, pLUXcadBp, pLUXcadCp, pLUXcbpAp, pLUXddlAp, pLUXgadAp, pLUXgadBp, pLUXgadCp, pLUXgadWp, pLUXglcVp, pLUXiraPp, pLUXpagPp, pLUXproVp, and pLUXslpp (Table 1), were introduced into $\Delta h n s \Delta h h a \Delta y d g T$ strains harbouring the hns plasmids (pQE80Lhns, pQE80Lhns-I70A, and pQE80Lhns-L75A) or an empty plasmid (a vector pQE80L). Transformants were grown in M9-glucose medium with $10 \mu \mathrm{M} \mathrm{IPTG}$ at $37^{\circ} \mathrm{C}$, and then the promoter activity was calculated as described above.

\section{Recipes}

1. Competent E. coli $\mathrm{DH} 5 \alpha$

a. Inoculate a single colony of $E$. coli DH5a in $5 \mathrm{ml}$ of LB broth

b. Incubate the pre-cultures at $37^{\circ} \mathrm{C}$ with shaking for overnight

c. Dilute overnight pre-culture 100 -fold in $10 \mathrm{ml}$ of fresh LB broth 
d. Incubate the cultures at $37^{\circ} \mathrm{C}$ with shaking until mid-logarithmic phase

e. Transfer $10 \mathrm{ml}$ of culture to a sterile $50 \mathrm{ml}$ plastic tube (Iwaki)

f. Collect the cells by centrifugation $\left(2,300 \times g, 4^{\circ} \mathrm{C}, 10 \mathrm{~min}\right)$

g. Suspend the cells in $10 \mathrm{ml}$ of cold $0.1 \mathrm{M} \mathrm{CaCl}_{2}$

h. Incubate the cells on ice for $30 \mathrm{~min}$.

i. Collect the cells by centrifugation $\left(2,300 \times \mathrm{g}, 4^{\circ} \mathrm{C}, 10 \mathrm{~min}\right)$

j. Suspend the cells in $1 \mathrm{ml}$ of cold $0.1 \mathrm{M} \mathrm{CaCl}_{2}$

k. Use a part $(\sim 100 \mu \mathrm{l})$ of the resuspended E. coli DH5a for transformation

2. $50 \mathrm{mg} / \mathrm{ml}$ kanamycin
a. Dissolve $0.5 \mathrm{~g}$ of kanamycin monosulfate (Nacalai Tesque) in $10 \mathrm{ml}$ of distilled water
b. Sterilize the solution by filtration with $0.22 \mu \mathrm{m}$ filter (Advantec)
C. Store the sterilized solution at $4{ }^{\circ} \mathrm{C}$

3. LB broth
a. Dissolve $10 \mathrm{~g}$ of Bacto ${ }^{\mathrm{TM}}$ tryptone, $5 \mathrm{~g}$ of Bacto $^{\mathrm{TM}}$ yeast extract, and $10 \mathrm{~g}$ of $\mathrm{NaCl}$ in $800 \mathrm{ml}$ of distilled water
b. Adjust $\mathrm{pH}$ to 7.5 with $\mathrm{NaOH}$
C. Adjust volume to $1 \mathrm{~L}$ with distilled water
d. Autoclave the solution (set $121^{\circ} \mathrm{C}$ and 20 min in LSX-500)
e. Store the autoclaved LB broth at room temperature

4. LB agar with $50 \mu \mathrm{g} / \mathrm{ml}$ kanamycin
a. Dissolve $7.5 \mathrm{~g}$ of agar in $1 \mathrm{~L}$ of LB broth
b. Autoclave (set $121^{\circ} \mathrm{C}$ and $20 \mathrm{~min}$ in LSX-500)
c. Add kanamycin after cooling in the final concentration of $50 \mu \mathrm{g} / \mathrm{ml}$
d. Pour the media into Petri dishes, and then harden LB agar by cooling at room temperature
e. Store the $\mathrm{LB}$ agar at $4{ }^{\circ} \mathrm{C}$

5. $125 \mathrm{mM}$ EDTA
a. Dilute $0.5 \mathrm{M}$ EDTA (Nacalai Tesque) to $125 \mathrm{mM}$ with a sterile water
b. Store at room temperature

6. $70 \%$ ethanol
a. Dilute ethanol (Nacalai Tesque) to $70 \%$ with a sterile water
b. Store at room temperature

7. M9-Glucose medium (to autoclave, set $121^{\circ} \mathrm{C}$ and $20 \mathrm{~min}$ in LSX-500)

a. For $5 x \mathrm{M} 9$ salt $\left(-\mathrm{NH}_{4} \mathrm{Cl}_{2}\right)$, dissolve $75 \mathrm{~g}$ of $\mathrm{Na}_{2} \mathrm{HPO}_{4} \cdot 12 \mathrm{H}_{2} \mathrm{O}, 15 \mathrm{~g}$ of $\mathrm{KH}_{2} \mathrm{PO}_{4}$ and $0.25 \mathrm{~g}$ of $\mathrm{NaCl}$ in $1 \mathrm{~L}$ distilled water, and then the dissolved solution was autoclaved

b. Prepare the autoclaved following solutions: $2 \mathrm{M} \mathrm{NH}_{4} \mathrm{Cl}_{2}, 1 \mathrm{M} \mathrm{MgCl}_{2}, 0.25 \mathrm{M} \mathrm{K}_{2} \mathrm{SO}_{4}, 10 \mathrm{mM}$ $\mathrm{CaCl}_{2}$

c. Sterilize the $1 \mathrm{M}$ glucose by filtration $(0.22 \mu \mathrm{m}$ filter $)$

d. Add $200 \mathrm{ml}$ of $5 \times \mathrm{M} 9$ salt $\left(-\mathrm{NH}_{4} \mathrm{Cl}_{2}\right), 10 \mathrm{ml}$ of $2 \mathrm{M} \mathrm{NH}_{4} \mathrm{Cl}_{2}, 1 \mathrm{ml}$ of $1 \mathrm{M} \mathrm{MgCl}_{2}, 1 \mathrm{ml}$ of $0.25 \mathrm{M}$ $\mathrm{K}_{2} \mathrm{SO}_{4}, 10 \mathrm{ml}$ of $10 \mathrm{mM} \mathrm{CaCl}, 10 \mathrm{ml}$ of $1 \mathrm{M}$ Glucose into $768 \mathrm{ml}$ of autoclaved distilled 
Please cite this article as: Yamanaka et. al., (2020). Measurement of the Promoter Activity in Escherichia coli by Using a Luciferase Reporter,Bio-protocol

water

e. Store the sterilized M9-glucouse medium at room temperature

\section{Acknowledgments}

We are extremely grateful to Prof. Akira Ishihama, Hosei Univeristy, for his comments and editing of the manuscript. We gratefully acknowledge Dr. Parisa Zangoui Nejad Chahkootahi (National University of Singapore) and Dr. Kyle David Buchan (National University of Singapore) for proofreading of the manuscript. The pLUX vector was a gift from Dr. Peter A. Lund, University of Birmingham. The E. coli $\Delta h n s \Delta h h a \Delta y d g T$ strain was a gift from Dr. Taku Oshima, Toyama Prefectural University. We thank Eri Arita, Kanako Hasegawa, Sho Watarai, Hideko Nakagawa, and Sato Fukunaga from Hosei university for technical assistance.

This protocol was adapted from Burton et al. (2010) for the method to luciferase reporter assay and modified from Yamanaka et al. (2018) for the method to high-throughput assay.

\section{Competing interests}

The authors declare no conflicts of interest.

\section{References}

1. Bjarnason, J., Southward, C. M. and Surette, M. G. (2003). Genomic profiling of iron-responsive genes in Salmonella enterica serovar typhimurium by high-throughput screening of a random promoter library. J Bacteriol 185(16): 4973-4982.

2. Burton, N. A., Johnson, M. D., Antczak, P., Robinson, A. and Lund, P. A. (2010). Novel aspects of the acid response network of $E$. coli K-12 are revealed by a study of transcriptional dynamics. J Mol Biol 401(5): 726-742.

3. Meighen, E. A. (1991). Molecular biology of bacterial bioluminescence. Microbiol Rev 55(1): 123-142.

4. Nozaki, S. and Niki, H. (2019). Exonuclease III (XthA) enforces in vivo DNA cloning of Escherichia coli to create cohesive ends. J Bacteriol 201(5): pii: e00660-18.

5. Ueda, T., Takahashi, H., Uyar, E., Ishikawa, S., Ogasawara, N. and Oshima, T. (2013). Functions of the Hha and YdgT proteins in transcriptional silencing by the nucleoid proteins, $\mathrm{H}$ NS and StpA, in Escherichia coli. DNA Res 20(3): 263-271.

6. Yamanaka, Y., Oshima, T., Ishihama, A., and Yamamoto, K. (2014). Characterization of the YdeO regulon in Escherichia coli. PLOS ONE 9: e111962.

7. Yamanaka, Y., Winardhi, R. S., Yamauchi, E., Nishiyama, S. I., Sowa, Y., Yan, J., Kawagishi, I., Ishihama, A. and Yamamoto, K. (2018). Dimerization site 2 of the bacterial DNA-binding 
Please cite this article as: Yamanaka et. al., (2020). Measurement of the Promoter Activity in Escherichia coli by Using a Luciferase Reporter,Bio-protocol 10 (2): e3500. DOI: 10.21769/BioProtoc.3500.

protein $\mathrm{H}-\mathrm{NS}$ is required for gene silencing and stiffened nucleoprotein filament formation. $J$ Biol

Chem 293(24): 9496-9505. 